\title{
An Analysis of Comprehension Performance of Sudanese EFL Students
}

\author{
Mahgoub Dafalla Ahmed ${ }^{1}$ \\ ${ }^{1}$ Department of English \& Translation, Faculty of Science \& Arts, Khulais Branch, University of Jeddah, Jeddah, \\ Saudi Arabia \\ Correspondence: Mahgoub Dafalla Ahmed, Department of English \& Translation, Faculty of Science \& Arts, \\ Khulais Branch, University of Jeddah, 21921 Jeddah, P.O. Box 355, Saudi Arabia. Tel: 966-534-053-521. E-mail: \\ mahgoub7700@hotmail.com
}

Received: April 9, 2015 Accepted: May 11, 2015 Online Published: June 29, 2015

doi:10.5539/elt.v8n7p133 URL: http://dx.doi.org/10.5539/elt.v8n7p133

\begin{abstract}
This study examines the Sudanese EFL students' comprehension performance and measures the differences in their performance according to gender. After one semester of participation in extensive reading, 300 secondary school students from 15 schools in the state of Khartoum are randomly selected for the study. A comprehension test followed by a questionnaire on how they think while completing the test are used as instruments. The study's results reveal an average performance and statistically significant differences in the background, explicit, vocabulary and general understanding questions of the reading comprehension. However, these significant differences are not found in the implicit and the interpretation questions and in using lower and higher levels of processing skills. In addition, significant differences have appeared between males and females students in the background, explicit, implicit and interpretation, and vocabulary questions, but not found in the general understanding questions. Then, findings are discussed and recommendations are provided. Drawing on these findings the study, for example, recommends that good applications of techniques and procedures of teaching EFL reading might be a viable intervention for improving students' performance in EFL reading.
\end{abstract}

Keywords: comprehension performance, comprehension reading, comprehension test, EFL students, reading skills, performance

\section{Introduction}

The situation of reading classes at Sudanese secondary schools and the complaints from students has obliged the researcher as an ELT practitioner to propose this study. Specially, no one can neglect the importance of reading and its necessity to successfulness in learning. Much work is done on reading EFL in general and reading strategies and reading skills, which are adopted by successful and unsuccessful learners in particular. Investigating lower and higher level of processing skills has occupied many efforts, also. To contribute in this field one of the researcher's aims from this study is to measure the levels of processing skills employed by Sudanese secondary schools students in EFL reading. However, his main aims are to examine the Sudanese EFL students' comprehension performance and measure the differences in their performance according to gender. The researcher has selected randomly 300 secondary school students from 15 schools in the state of Khartoum. At the end of the study, he hopes to reach to results that can help to improve EFL reading in the Sudan and create better and effective readers.

\section{Literature Review}

\subsection{Theoretical Background}

Reading is considered by many researchers as a vital skill in learning, and reading ability is necessary for any learner searching for success. Due to the complexity of the reading processes, readers have to benefit from the background knowledge, linguistic knowledge, encyclopedic knowledge, contextual knowledge, etc. they possess and all their skills and strategies of reading in order to comprehend the meanings of the text. Crystal (1987, p. 209) notes,

"The field of reading research would not seem to be a particularly promising or attractive one. It is, however, an area that has attracted many investigators, partly by virtue of its very complexity, and partly because any 
solutions to the problem of how we read would have immediate application in areas of high social concern".

Reading have had many shifts and transitions during time and many approaches and theories have been proposed beginning from focusing on the printed text to the cognitive and metacognitive theories. Therefore, many researchers have considered reading process and have different views about it. In Goodman's (1967) view of reading, readers benefit from their background knowledge when they read. However, Rumelhart (1977) has emphasized on the role of schemata in reading. Anderson and Pearson (1984) think reading require readers to interpret the new information and assimilate them with the information already exist at their memories. Bamford and Day (1998) refer to reading as constructing meaning from a written text. Mikulecky (1990) do not separate between reading and comprehension. Nattal (1983), on the other hand, is convinced that reading happens spontaneously, not by teaching. For Aebersold and Field (1997), reading occurs from looking at the text and assigning meaning from its written symbols. Eskey (1988) thinks the reader needs a complete interaction with the text to be comprehend. For Eskey, reading in L2 demands more cognitive effort.

\subsection{Lower or Higher Levels of Processing Skills?}

Many researchers in second language reading such as Carrel (1988) and Eskey (1988) deal with the lower level (Bottom up) and the higher level (top down) processing skills of reading. However, other researchers such as Gagne et al. (1993) have divided reading processes into more subgroups including the bottom up level of processing skills and higher level of processing skills.

In addition, reading comprehension's studies vary in evaluating which processing skill is better to be adopted by students. After making much research on skilled and less-skilled readers, some studies prefer focusing on engaging students in bottom-up, some on top-down processing skills. However, good readers always use interactive reading, which integrates elements of both levels of processing. In fact, most readers begin reading by using top-down in reading strategies until they face a problem like encountering an unknown word, then they shift to bottom-up reading strategies. They slow down to decode the new word.

\subsection{Competence and Performance in EFL Reading Comprehension}

Fromkin and Rodman (1981) refer to reading performance as "how" the language is used by someone. In order to maximize the validity of performance, students and teachers need to be aware reading in a foreign language needs certain techniques and strategies to be mastered. Nation (2009, p. 149) describes a number of them for improving readers' reading skills. He emphasizes that the techniques and strategies should not be seen as isolated activities but as means of bringing meaning into practice. To be effective and efficient readers, EFL students have to tackle all the following elements of competences: Phonological competence: knowledge of sounds and sound combinations; syntactic competence: knowledge about the possible syntactic combinations; semantic competence: knowledge about the meanings of words, phrases and sentences; lexical competence: having extensive amount of words; morphological competence: knowledge of word formation or word structure; communicative competence: knowledge about the social, pragmatic and contextual characteristics of a language; grammatical competence (linguistic competence): knowledge of the use of different functioning rules of the system of the language; sociolinguistic competence: knowledge of producing sentences according to the communicative situations i.e., knowing when, where and whom to say things; discursive competence: knowledge of determining different types of discourse; and strategic competence: knowledge to maintain communication i.e., strategies language users have to understand others.

\section{Hypotheses}

1) There is no statistically significant difference in the mean comprehension performance in EFL reading among Sudanese secondary school students.

2) There is no statistically significant difference between the mean comprehension performance in EFL reading of Sudanese secondary school males and females students.

\section{Method}

\subsection{Subjects and Setting}

To identify the subjects for the study, the data was collected from 15 secondary geographical schools in the state of Khartoum. Participants of the study were 300 students in total, 160 male and 140 female (20 students from each school). They were Sudanese EFL learners at secondary level, at their $3^{\text {rd }}$ grade. At the time of testing, they had studied English for seven years and they were about to finish the SPINE (Note 1) series. Their ages ranged from 16 to 18 . 


\subsection{Measuring Instruments}

The study used both quantitative and qualitative data analysis. The students took a 25 -item reading comprehension test, followed by a questionnaire on how they thought while completing the test.

\subsubsection{The Test}

Although the criticism it faces as inappropriate measure of students' academic competence, the test used was a traditional standardized objective achievement test. The researcher selected this type of test of reading to focus on the integration of lower level and higher level of skills. The students were given a reading passage preceded by background questions and followed by different other kinds of questions (see Appendix A). The reading text was adapted from the online article, "Two types of Input Modification and EFL Reading Comprehension: Simplification versus Elaboration" by Sun-Young Oh (2001). It was the elaborated form, which was used in his study to investigate the relative effects of input modification and elaboration on Korean high school students' EFL reading comprehension. The text was intended to achieve the designed objective in that it was long to some extent with about 250 words, new information and vocabulary, included clues, defining context and relative clauses. The researcher prepared a set of 25 written questions to be answered before and after reading the text. They were five different types of questions (A - E) measuring both bottom-up and top-down skills including background questions, literal (explicit) questions, implicit and interpretation questions, vocabulary questions and questions on general or overall understanding. They consisted of general, specific and inferential comprehension questions. Through answering the questions, the researcher could check and measure progress in such important elements of EFL reading. Without any preparation, the participants were asked to read the text silently and answer the questions.

\subsubsection{The Questionnaire}

For the purpose of the study, the questionnaire was designed to be answered by the students who did the test (see Appendix B). Information obtained covered the two main parts of the processing levels of skills: bottom-up and top-down. It was designed according to the options of the Likert five - point scale ("strongly agree", "agree", "somewhat", "disagree", and "strongly disagree"). Participant students were asked to give their personal information, then, provided answers on how they thought while completing the test. The questionnaire had 22 statements.

\subsubsection{Correlations \& Reliability}

To measure the reliability of the comprehension test, the researcher chose the "test-retest" reliability to find out whether the questions were related to one another and measure the same thing or not. The students answered the same test twice. The interval time between the two tests was two weeks. Forty-four students did the first test. Forty-five students did the second. Forty students did the two tests. The correlations of the scores of the two comprehension tests was (0.75) according to Pearson correlations coefficients. On the other hand, the questionnaire was given to many faculty members and classroom practitioners. Twenty teachers were expected to fill it in; however, only 15 handed in their copies. Therefore, the reliability was calculated from the 15 copies using the split-half method. To apply this method, first the questions were divided into two similar parts. Since the items were homogenous, all odd-numbered items constituted one half and even-numbered items constituted the other half. Then, the scores of the subjects on the two halves of the test were correlated. The reported questions reliability of (0.79) using Guttmann's prophecy formula showed that this instrument was highly reliable.

\subsubsection{Procedures}

Dealing with the target group, 20 students (randomly selected from each class) received instructions from the researcher in the remedial setting. The reading comprehension test was given to students to be read silently. The students wrote their answers to the written question. They finished the test in a single session that lasted for around 45 minutes. Instructors provided their assistance to students answering their questions about the comprehension questions, but not in answering them. Each student finished doing the test, the instructor handed him the questionnaire to be filled out.

\section{Analysis, Results \& Discussions}

The focus of this part is to analyze, discuss and state the results and findings. The data to be analyzed and discussed was gathered by both instruments (the students' test and the questionnaire). Therefore, the analysis, discussions and results of the data collected and used were treated by virtue of these two instruments. Then, different statistical procedures were done. Statistical results in relation to hypotheses were drawn and discussed. Each hypothesis was restated and followed by an examination of the statistical results relating to it. 
1) Hypothesis One: There is no statistically significant difference in the mean comprehension performance in EFL reading among Sudanese secondary school students

To test this hypothesis, the scores of students in the reading comprehension test were obtained. Their performances were evaluated in five areas: background questions; explicit questions; implicit and interpretation; vocabulary; and general understanding. Since the total mark of the test was 25 , the passing mark was regarded as 12.5. To see the performances of students in EFL reading and check whether this assumption was valid or not, the one-sample $t$-test was used. The mean scores, standard deviations and the P-values were calculated and their results were shown in Table 1.

Table 1. Average performance in EFL reading test

\begin{tabular}{llllllllll}
\hline Area & $\mathrm{N}$ & $\begin{array}{l}\text { Full } \\
\text { Mark }\end{array}$ & $\begin{array}{l}\text { Pass } \\
\text { Mark }\end{array}$ & Success & $\mathrm{M}$ & SD. & C.V & T-value & P-value \\
\hline Background & 300 & 6 & 3 & $68 \%$ & 2.50 & 2.00 & $57.1 \%$ & 4.20 & $0.000^{*}$ \\
Explicit questions & 300 & 5 & 3 & $41 \%$ & 2.38 & 1.45 & $60.9 \%$ & 7.45 & $0.000^{*}$ \\
Implicit \& interpretation & 300 & 6 & 3 & $69 \%$ & 3.19 & 1.37 & $42.9 \%$ & 2.49 & $0.013^{*}$ \\
Vocabulary & 300 & 5 & 3 & $52 \%$ & 2.49 & 1.23 & $49.4 \%$ & 7.21 & $0.000^{*}$ \\
General understanding & 300 & 3 & 2 & $56 \%$ & 1.40 & 0.91 & $65.0 \%$ & 11.39 & $0.000^{*}$ \\
Total & 300 & 25 & 13 & $56 \%$ & 12.89 & 5.10 & $39.6 \%$ & 0.35 & 0.726 \\
\hline
\end{tabular}

* Difference is significant at or less than 0.05 level of significance.

The results of the one-sample $t$-test showed significant differences in the background $(\mathrm{M}=2.50, \mathrm{SD}=2.00, \mathrm{~N}=$ $300)$, explicit $(\mathrm{M}=2.38, \mathrm{SD}=1.45, \mathrm{~N}=300)$, vocabulary $(\mathrm{M}=2.49, \mathrm{SD}=1.23, \mathrm{~N}=300)$ and the general understanding questions $(\mathrm{M}=1.40, \mathrm{SD}=0.91, \mathrm{~N}=300)$. Their T-values of 4.20, 7.45, 7.21 and 11.39 respectively with a common P-value of 0.000 which was below the level of significance 0.05 indicated that these groups were significantly different. When their mean scores were compared with the passing mark, the averages were found significantly below the passing mark. On the other hand, the results showed there was no significant difference in the part of the implicit and interpretation questions $(\mathrm{M}=3.19, \mathrm{SD}=1.37, \mathrm{~N}=300)$. Its t-value was 1.49 with a P-value of 0.013 was larger than 0.05 , the level of significance. When its mean score was compared with the passing mark, the average was found significantly above the passing mark. In addition, the coefficient of variation (C.V) showed that there were variations of performances in all sections of the test. The highest variation was in the general understanding section (65\%) followed with explicit questions section $(60.9 \%)$, and the least variation was obtained in implicit and interpretation section $(42.9 \%)$.

These results indicated that the performances of students differed in some sections and did not differ in others and generally tallying to an average. Therefore, these results did not confirm or reject the hypothesis that there was no statistically significant difference between the mean comprehension performances in EFL reading of Sudanese secondary school students in some parts and rejected the hypothesis in others.

Furthermore, although explicit questions were always simpler than the implicit and the interpretation ones, the results of the comprehension test showed the highest percentage of success was gained in the implicit and interpretation questions (69\%) and background questions (68\%), however, the learners failed in giving correct responses to the explicit questions (41\%). These results indicated that the students tended to possess abilities in top-down skills rather than bottom-up skills. Figure (1) showed the average performance of the students in all sections of the EFL reading test. 


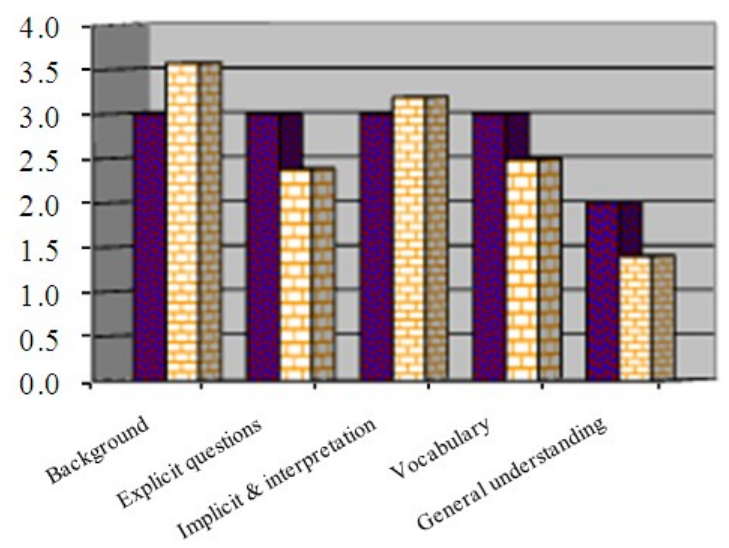

- Pass mark $\square_{\text {Mean score }}$

Figure 1. The average performance of the students in EFL reading test

Concerning the questionnaire that was introduced to students to express their thought while they were answering the comprehension reading questions, the $t$-test for independent samples was used. The mean scores and standard deviations were calculated in Tables 2 and 3.

Table 2. The group statistics of the questionnaire (bottom-up statements)

\begin{tabular}{llllll}
\hline & Sex & N & Mean & Std. Deviation & Std. Error Mean \\
\hline \multirow{2}{*}{ Bottom-up S1 } & Male & 160 & 3.18 & 1.154 & 0.091 \\
& Female & 140 & 3.44 & 1.054 & 0.089 \\
Bottom-up S2 & Male & 160 & 3.29 & 1.107 & 0.088 \\
& Female & 140 & 3.33 & 1.042 & 0.088 \\
Bottom-up S3 & Male & 160 & 3.32 & 1.030 & 0.081 \\
& Female & 140 & 3.38 & 1.049 & 0.089 \\
Bottom-up S4 & Male & 160 & 2.71 & 1.090 & 0.086 \\
& Female & 140 & 2.56 & 1.041 & 0.088 \\
Bottom-up S5 & Male & 160 & 2.97 & 0.921 & 0.073 \\
& Female & 140 & 3.02 & 1.007 & 0.085 \\
Bottom-up S6 & Male & 160 & 2.61 & 0.972 & 0.077 \\
& Female & 140 & 2.39 & 1.043 & 0.088 \\
Bottom-up S7 & Male & 160 & 3.19 & 1.029 & 0.081 \\
& Female & 140 & 3.25 & 1.120 & 0.095 \\
\hline
\end{tabular}


Table 3. The group statistics of the questionnaire (top-down statements)

\begin{tabular}{|c|c|c|c|c|c|}
\hline & Sex & $\mathrm{N}$ & Mean & Std. Deviation & Std. Error Mean \\
\hline \multirow{2}{*}{ Top-down S8 } & Male & 160 & 3.20 & 1.039 & 0.082 \\
\hline & Female & 140 & 3.30 & 1.049 & 0.089 \\
\hline \multirow{2}{*}{ Top-down S9 } & Male & 160 & 3.32 & 0.948 & 0.075 \\
\hline & Female & 140 & 3.16 & 1.101 & 0.093 \\
\hline \multirow{2}{*}{ Top-down S10 } & Male & 160 & 3.02 & 1.084 & 0.086 \\
\hline & Female & 140 & 3.07 & 1.070 & 0.090 \\
\hline \multirow{2}{*}{ Top-down S11 } & Male & 160 & 3.18 & 1.063 & 0.084 \\
\hline & Female & 140 & 3.03 & 1.112 & 0.094 \\
\hline \multirow{2}{*}{ Top-down S12 } & Male & 160 & 2.98 & 1.055 & 0.083 \\
\hline & Female & 140 & 3.09 & 1.065 & 0.090 \\
\hline \multirow{2}{*}{ Top-down S13 } & Male & 160 & 3.20 & 1.080 & 0.085 \\
\hline & Female & 140 & 3.34 & 1.084 & 0.092 \\
\hline \multirow{2}{*}{ Top-down S14 } & Male & 160 & 3.10 & 1.134 & 0.090 \\
\hline & Female & 140 & 3.15 & 1.079 & 0.091 \\
\hline \multirow{2}{*}{ Top-down S15 } & Male & 160 & 3.06 & 1.114 & 0.088 \\
\hline & Female & 140 & 2.94 & 1.098 & 0.093 \\
\hline \multirow{2}{*}{ Top-down S16 } & Male & 160 & 3.16 & 1.104 & 0.087 \\
\hline & Female & 140 & 3.04 & 1.045 & 0.088 \\
\hline \multirow{2}{*}{ Top-down S17 } & Male & 160 & 2.97 & 1.129 & 0.089 \\
\hline & Female & 140 & 3.29 & 1.232 & 0.104 \\
\hline \multirow{2}{*}{ Top-down S18 } & Male & 160 & 2.77 & 1.250 & 0.099 \\
\hline & Female & 140 & 2.98 & 1.266 & 0.107 \\
\hline \multirow{2}{*}{ Top-down S19 } & Male & 160 & 2.92 & 1.149 & 0.091 \\
\hline & Female & 140 & 2.81 & 1.199 & 0.101 \\
\hline \multirow{2}{*}{ Top-down S20 } & Male & 160 & 2.67 & 1.258 & 0.099 \\
\hline & Female & 140 & 2.61 & 1.160 & 0.098 \\
\hline \multirow{2}{*}{ Top-down S21 } & Male & 160 & 2.67 & 1.282 & 0.101 \\
\hline & Female & 140 & 2.70 & 1.268 & 0.107 \\
\hline \multirow{2}{*}{ Top-down S22 } & Male & 160 & 2.79 & 1.264 & 0.100 \\
\hline & Female & 140 & 2.72 & 1.212 & 0.102 \\
\hline
\end{tabular}

The results showed no significant differences found between the mean scores of all the variants. When looking at Tables 4 and 5 for the independent samples test, we can check the assumption of equal variances. In addition, when looking at Levine's test for equality of variances, we can determine the scores of all variants of the groups. 
Table 4. Independent samples $t$-test (questionnaire's bottom-up statements)

\begin{tabular}{|c|c|c|c|c|c|c|c|c|c|c|}
\hline & & \multicolumn{2}{|c|}{ Equality of Variances t-test Ior Equanty or Means } & \multicolumn{7}{|c|}{ t-test for Equality of Means } \\
\hline & & \multirow{3}{*}{$\mathrm{F}$} & \multirow{3}{*}{ Sig. } & \multirow{3}{*}{$\mathrm{t}$} & \multirow{3}{*}{ df } & \multirow{3}{*}{$\begin{array}{l}\text { Sig. } \\
\text { (2-tailed) }\end{array}$} & \multirow{3}{*}{$\begin{array}{l}\text { Mean } \\
\text { Difference }\end{array}$} & \multirow{3}{*}{$\begin{array}{l}\text { Std. Error } \\
\text { Difference }\end{array}$} & \multirow{2}{*}{\multicolumn{2}{|c|}{$\begin{array}{l}95 \% \text { Confidence } \\
\text { Interval for the } \\
\text { Difference }\end{array}$}} \\
\hline & & & & & & & & & & \\
\hline & & & & & & & & & Lower & Upper \\
\hline \multirow{2}{*}{$\begin{array}{l}\text { Bottom-up } \\
\text { S1 }\end{array}$} & $\begin{array}{l}\text { Equal variances } \\
\text { assumed }\end{array}$ & \multirow{2}{*}{0.748} & \multirow{2}{*}{0.388} & -2.039 & 298 & 0.042 & -0.262 & 0.128 & -0.514 & -0.009 \\
\hline & $\begin{array}{l}\text { Equal variances } \\
\text { not assumed }\end{array}$ & & & -2.051 & 297.431 & 0.041 & -0.262 & 0.128 & -0.513 & -0.011 \\
\hline \multirow{2}{*}{$\begin{array}{l}\text { Bottom-up } \\
\text { S2 }\end{array}$} & $\begin{array}{l}\text { Equal variances } \\
\text { assumed }\end{array}$ & \multirow[b]{2}{*}{0.610} & \multirow{2}{*}{0.435} & -0.330 & 298 & 0.742 & -0.041 & 0.125 & -0.286 & 0.204 \\
\hline & $\begin{array}{l}\text { Equal variances } \\
\text { not assumed }\end{array}$ & & & -0.331 & 296.394 & 0.741 & -0.041 & 0.124 & -0.285 & 0.203 \\
\hline \multirow{2}{*}{$\begin{array}{l}\text { Bottom-up } \\
\text { S3 }\end{array}$} & $\begin{array}{l}\text { Equal variances } \\
\text { assumed }\end{array}$ & \multirow[b]{2}{*}{0.107} & \multirow[b]{2}{*}{0.744} & -0.498 & 298 & 0.619 & -0.060 & 0.120 & -0.296 & 0.177 \\
\hline & $\begin{array}{l}\text { Equal variances } \\
\text { not assumed }\end{array}$ & & & -0.497 & 291.266 & 0.620 & -0.060 & 0.120 & -0.297 & 0.177 \\
\hline \multirow{2}{*}{$\begin{array}{l}\text { Bottom-up } \\
\text { S4 }\end{array}$} & $\begin{array}{l}\text { Equal variances } \\
\text { assumed }\end{array}$ & \multirow{2}{*}{0.257} & \multirow{2}{*}{0.612} & 1.258 & 298 & 0.209 & 0.155 & 0.123 & -0.088 & 0.398 \\
\hline & $\begin{array}{l}\text { Equal variances } \\
\text { not assumed }\end{array}$ & & & 1.262 & 295.703 & 0.208 & 0.155 & 0.123 & -0.087 & 0.398 \\
\hline \multirow{2}{*}{$\begin{array}{l}\text { Bottom-up } \\
\text { S5 }\end{array}$} & $\begin{array}{l}\text { Equal variances } \\
\text { assumed }\end{array}$ & \multirow{2}{*}{0.993} & \multirow{2}{*}{0.320} & -0.473 & 298 & 0.636 & -0.053 & 0.111 & -0.272 & 0.166 \\
\hline & $\begin{array}{l}\text { Equal variances } \\
\text { not assumed }\end{array}$ & & & -0.470 & 283.951 & 0.638 & -0.053 & 0.112 & -0.273 & 0.168 \\
\hline \multirow{2}{*}{$\begin{array}{l}\text { Bottom-up } \\
\text { S6 }\end{array}$} & $\begin{array}{l}\text { Equal variances } \\
\text { assumed }\end{array}$ & \multirow{2}{*}{1.057} & \multirow{2}{*}{0.305} & 1.895 & 298 & 0.059 & 0.221 & 0.116 & -0.008 & 0.450 \\
\hline & $\begin{array}{l}\text { Equal variances } \\
\text { not assumed }\end{array}$ & & & 1.886 & 286.100 & 0.060 & 0.221 & 0.117 & -0.010 & 0.451 \\
\hline \multirow{2}{*}{$\begin{array}{l}\text { Bottom-up } \\
\text { S7 }\end{array}$} & $\begin{array}{l}\text { Equal variances } \\
\text { assumed }\end{array}$ & \multirow{2}{*}{3.128} & \multirow{2}{*}{0.078} & -0.504 & 298 & 0.615 & -0.063 & 1.124 & -0.307 & 0.182 \\
\hline & $\begin{array}{l}\text { Equal variances } \\
\text { not assumed }\end{array}$ & & & -0.501 & 284.528 & 0.617 & -0.063 & 0.125 & -0.308 & 0.183 \\
\hline
\end{tabular}

Table 5. Independent samples $t$-test (questionnaire's top-down statements)

\begin{tabular}{|c|c|c|c|c|c|c|c|c|c|c|}
\hline & & $\begin{array}{l}\text { Levine' } \\
\text { Equalit }\end{array}$ & $\begin{array}{l}\text { Test } \\
\text { Varian }\end{array}$ & \multicolumn{7}{|c|}{ t-test for Equality of Means } \\
\hline & & \multirow[t]{2}{*}{$\mathrm{F}$} & \multirow[t]{2}{*}{ Sig. } & \multirow[t]{2}{*}{$\mathrm{t}$} & \multirow[t]{2}{*}{$\mathrm{df}$} & \multirow[t]{2}{*}{$\begin{array}{l}\text { Sig. } \\
\text { (2-tailed) }\end{array}$} & \multirow[t]{2}{*}{$\begin{array}{l}\text { Mean } \\
\text { Difference }\end{array}$} & \multirow[t]{2}{*}{$\begin{array}{l}\text { Std. Error } \\
\text { Difference }\end{array}$} & \multicolumn{2}{|c|}{$\begin{array}{l}95 \% \text { Confidence } \\
\text { Interval for the } \\
\text { Difference }\end{array}$} \\
\hline & & & & & & & & & Lower & Upper \\
\hline \multirow{2}{*}{$\begin{array}{l}\text { Top-down } \\
\text { S8 }\end{array}$} & $\begin{array}{l}\text { Equal variances } \\
\text { assumed }\end{array}$ & \multirow{2}{*}{0.263} & \multirow{2}{*}{0.609} & -1.065 & 0.298 & 0.288 & -0.129 & 0.121 & -0.366 & 0.109 \\
\hline & $\begin{array}{l}\text { Equal variances } \\
\text { not assumed }\end{array}$ & & & -1.064 & 291.985 & 0.288 & -0.129 & 0.121 & -0.366 & 0.109 \\
\hline \multirow{2}{*}{$\begin{array}{l}\text { Top-down } \\
\text { S9 }\end{array}$} & $\begin{array}{l}\text { Equal variances } \\
\text { assumed }\end{array}$ & \multirow{2}{*}{1.879} & \multirow{2}{*}{0.171} & 1.366 & 0.298 & 0.173 & 0.162 & 0.118 & -0.071 & 0.394 \\
\hline & $\begin{array}{l}\text { Equal variances } \\
\text { not assumed }\end{array}$ & & & 1.353 & 276.126 & 0.177 & 0.162 & 0.119 & -0.074 & 0.397 \\
\hline
\end{tabular}




\begin{tabular}{|c|c|c|c|c|c|c|c|c|c|c|}
\hline \multirow{2}{*}{$\begin{array}{l}\text { Top-down } \\
\text { S10 }\end{array}$} & $\begin{array}{l}\text { Equal variances } \\
\text { assumed }\end{array}$ & \multirow[b]{2}{*}{0.007} & \multirow[b]{2}{*}{0.931} & -0.422 & 0.298 & 0.673 & -0.053 & 0.125 & -0.298 & 0.193 \\
\hline & $\begin{array}{l}\text { Equal variances } \\
\text { not assumed }\end{array}$ & & & -0.423 & 293.682 & 0.673 & -0.053 & 0.125 & -0.298 & 0.193 \\
\hline \multirow{2}{*}{$\begin{array}{l}\text { Top-down } \\
\text { S11 }\end{array}$} & $\begin{array}{l}\text { Equal variances } \\
\text { assumed }\end{array}$ & \multirow[b]{2}{*}{0.003} & \multirow[b]{2}{*}{0.955} & 1.215 & 0.298 & 0.226 & 0.153 & 0.126 & -0.095 & 0.400 \\
\hline & $\begin{array}{l}\text { Equal variances } \\
\text { not assumed }\end{array}$ & & & 1.211 & 288.780 & 0.227 & 0.153 & 0.126 & -0.095 & 0.401 \\
\hline \multirow{2}{*}{$\begin{array}{l}\text { Top-down } \\
\text { S12 }\end{array}$} & $\begin{array}{l}\text { Equal variances } \\
\text { assumed }\end{array}$ & \multirow{2}{*}{0.641} & \multirow{2}{*}{0.424} & -0.910 & 0.298 & 0.364 & -0.112 & 0.123 & -0.353 & 0.130 \\
\hline & $\begin{array}{l}\text { Equal variances } \\
\text { not assumed }\end{array}$ & & & -0.909 & 291.947 & 0.364 & -0.112 & 0.123 & -0.353 & 0.130 \\
\hline \multirow{2}{*}{$\begin{array}{l}\text { Top-down } \\
\text { S13 }\end{array}$} & $\begin{array}{l}\text { Equal variances } \\
\text { assumed }\end{array}$ & \multirow{2}{*}{0.249} & \multirow{2}{*}{0.618} & -1.084 & 0.298 & 0.279 & -0.136 & 0.125 & -0.382 & 0.111 \\
\hline & $\begin{array}{l}\text { Equal variances } \\
\text { not assumed }\end{array}$ & & & -1.084 & 292.506 & 0.279 & -0.136 & 0.125 & -0.382 & 0.111 \\
\hline \multirow{2}{*}{$\begin{array}{l}\text { Top-down } \\
\text { S14 }\end{array}$} & $\begin{array}{l}\text { Equal variances } \\
\text { assumed }\end{array}$ & \multirow{2}{*}{0.154} & \multirow{2}{*}{0.695} & -0.390 & 0.298 & 0.697 & -0.050 & 0.28 & -0.302 & 0.202 \\
\hline & $\begin{array}{l}\text { Equal variances } \\
\text { not assumed }\end{array}$ & & & -0.391 & 295.883 & 0.696 & -0.050 & 0.128 & -0.302 & 0.202 \\
\hline \multirow{2}{*}{$\begin{array}{l}\text { Top-down } \\
\text { S15 }\end{array}$} & $\begin{array}{l}\text { Equal variances } \\
\text { assumed }\end{array}$ & \multirow{2}{*}{0.005} & \multirow{2}{*}{0.942} & -0.934 & 0.298 & 0.351 & 0.120 & 0.128 & -0.132 & 0.372 \\
\hline & $\begin{array}{l}\text { Equal variances } \\
\text { not assumed }\end{array}$ & & & -0.935 & 293.812 & 0.350 & 0.120 & 0.128 & -0.132 & 0.371 \\
\hline \multirow{2}{*}{$\begin{array}{l}\text { Top-down } \\
\text { S16 }\end{array}$} & $\begin{array}{l}\text { Equal variances } \\
\text { assumed }\end{array}$ & \multirow{2}{*}{1.170} & \multirow{2}{*}{0.280} & 0.960 & 0.298 & 0.338 & 0.120 & 0.125 & -0.126 & 0.365 \\
\hline & $\begin{array}{l}\text { Equal variances } \\
\text { not assumed }\end{array}$ & & & 0.964 & 296.151 & 0.336 & 0.120 & 0.124 & -0.125 & 0.364 \\
\hline \multirow{2}{*}{$\begin{array}{l}\text { Top-down } \\
\text { S17 }\end{array}$} & $\begin{array}{l}\text { Equal variances } \\
\text { assumed }\end{array}$ & \multirow{2}{*}{2.016} & \multirow{2}{*}{0.157} & -2.377 & 0.298 & 0.018 & -0.324 & 0.136 & -0.592 & -0.056 \\
\hline & $\begin{array}{l}\text { Equal variances } \\
\text { not assumed }\end{array}$ & & & -2.363 & 284.232 & 0.019 & -0.324 & 0.137 & -0594 & -0.054 \\
\hline \multirow{2}{*}{$\begin{array}{l}\text { Top-down } \\
\text { S18 }\end{array}$} & $\begin{array}{l}\text { Equal variances } \\
\text { assumed }\end{array}$ & \multirow{2}{*}{0.900} & \multirow{2}{*}{0.344} & -1.442 & 0.298 & 0.150 & -0.210 & 0.146 & -0.496 & 0.077 \\
\hline & $\begin{array}{l}\text { Equal variances } \\
\text { not assumed }\end{array}$ & & & -1.440 & 291.689 & 0.151 & -0.210 & 0.146 & -0.497 & 0.077 \\
\hline \multirow{2}{*}{$\begin{array}{l}\text { Top-down } \\
\text { S19 }\end{array}$} & $\begin{array}{l}\text { Equal variances } \\
\text { assumed }\end{array}$ & \multirow{2}{*}{0.556} & \multirow{2}{*}{0.456} & 0.822 & 0.298 & 0.411 & 0.112 & 0.136 & -0.155 & 0.379 \\
\hline & $\begin{array}{l}\text { Equal variances } \\
\text { not assumed }\end{array}$ & & & 0.820 & 289.019 & 0.413 & 0.112 & 0.136 & -0.156 & 0.379 \\
\hline \multirow{2}{*}{$\begin{array}{l}\text { Top-down } \\
\text { S20 }\end{array}$} & $\begin{array}{l}\text { Equal variances } \\
\text { assumed }\end{array}$ & 0.881 & 0.349 & 0.388 & 0.298 & 0.698 & 0.054 & 0.140 & -0.222 & 0.331 \\
\hline & $\begin{array}{l}\text { Equal variances } \\
\text { not assumed }\end{array}$ & & & 0.390 & 297.148 & 0.697 & 0.054 & 0.140 & -0.220 & 0.329 \\
\hline Top-down & $\begin{array}{l}\text { Equal variances } \\
\text { assumed }\end{array}$ & 0.089 & 0.766 & -0.212 & 0.298 & 0.832 & -0.031 & 0.148 & -0.322 & 0.259 \\
\hline S2I & $\begin{array}{l}\text { Equal variances } \\
\text { not assumed }\end{array}$ & & & -0.212 & 293.581 & 0.832 & -.0 .031 & 0.148 & -0.322 & 0.259 \\
\hline Top-down & $\begin{array}{l}\text { Equal variances } \\
\text { assumed }\end{array}$ & 0.180 & 0.672 & 0.504 & 0.298 & 0.615 & 0.072 & 0.144 & -0.210 & 0.355 \\
\hline $\mathrm{S} 22$ & $\begin{array}{l}\text { Equal variances } \\
\text { not assumed }\end{array}$ & & & 0.505 & 295.518 & 0.615 & 0.072 & 0.143 & -0.209 & 0.354 \\
\hline
\end{tabular}


The results of the independent $t$-test showed no statistically significant differences between all variants of the groups. They were all larger than 0.05 , indicating that there were no significant differences between the scores of all variants of the questionnaire. This means that the assumption of the equal variances was not violated and it was tenable.

\section{2) Hypothesis Tow: There is no statistically significant difference between the mean comprehension performance} in EFL reading of Sudanese secondary school males and females students

To test this hypothesis, the average scores of students in the comprehension test and the questionnaire were obtained. Concerning the comprehension test, the hypothesis was tested by using a $t$-test for independent samples. Table (6) showed the performances of male and female students in the comprehension test. The mean scores of male and female students in the five areas and the overall mark were compared.

Table 6. The average performance of male and female students in the comprehension test

\begin{tabular}{llllllll}
\hline Area & Sex & No. of cases & M & SD & C.V & T-value & P-value \\
\hline \multirow{2}{*}{ Background questions } & Male & 160 & 2.96 & 1.85 & 62.5 & \multirow{2}{*}{4.96} & \multirow{2}{*}{$0.001^{*}$} \\
& Female & 140 & 4.07 & 2.01 & 49.4 & & \\
Explicit questions & Male & 160 & 1.99 & 1.35 & 67.6 & \multirow{2}{*}{5.09} & $0.000^{*}$ \\
\multirow{2}{*}{ Implicit \& interpretation } & Female & 140 & 2.81 & 1.44 & 51.2 & & \\
questions & Male & 160 & 2.84 & 1.37 & 48.2 & \multirow{2}{*}{4.97} & $0.000^{*}$ \\
Vocabulary questions & Female & 140 & 3.60 & 1.25 & 34.6 & & \\
& Male & 160 & 2.20 & 1.19 & 54.1 & \multirow{2}{*}{4.52} & $0.000^{*}$ \\
General understanding & Female & 140 & 2.82 & 1.18 & 41.9 & & \\
& Male & 160 & 1.33 & 0.91 & 69.0 & \multirow{2}{*}{1.60} & 0.110 \\
& Female & 140 & 1.49 & 0.89 & 59.9 & & \\
& Male & 160 & 11.24 & 4.74 & 42.2 & \multirow{2}{*}{6.41} & $0.000^{*}$ \\
\hline
\end{tabular}

* Difference is significant at $5 \%$.

The results of the one-sample $t$-test showed significant differences in the background $(\mathrm{M}=4.07, \mathrm{SD}=2.01, \mathrm{~N}=$ $140)$, explicit $(\mathrm{M}=2.81, \mathrm{SD}=1.44, \mathrm{~N}=140)$, implicit and interpretation $(\mathrm{M}=3.60, \mathrm{SD}=1.25, \mathrm{~N}=140)$, and the vocabulary questions $(\mathrm{M}=2.82, \mathrm{SD}=1.18, \mathrm{~N}=140)$. Their T-values were of $4.96,5.09,4.97$ and 4.52 respectively with a common P-value of 0.000 which was below the level of significance 0.05 indicated that these groups were significantly different. On the other hand, the results showed there was no significant difference in the part of general understanding questions $(\mathrm{M}=1.33, \mathrm{SD}=0.91, \mathrm{~N}=160)$. Its t-value was 1.60 with a P-value of 0.110 was larger than 0.05 , the level of significance.

The results showed the average performance of female students was better than that of male in all areas of EFL reading test except in the general understanding section where there were no statistically significant differences between the scores of the two sexes. In general, comparing to male students, not only the performance of female students was better than their counterparts were but also was characterized by the small coefficient of variations (C.V). This result showed that the performance of female was more homogenous than that of male students. Figure 2 showed the average performance of male and female students in the EFL reading test. 


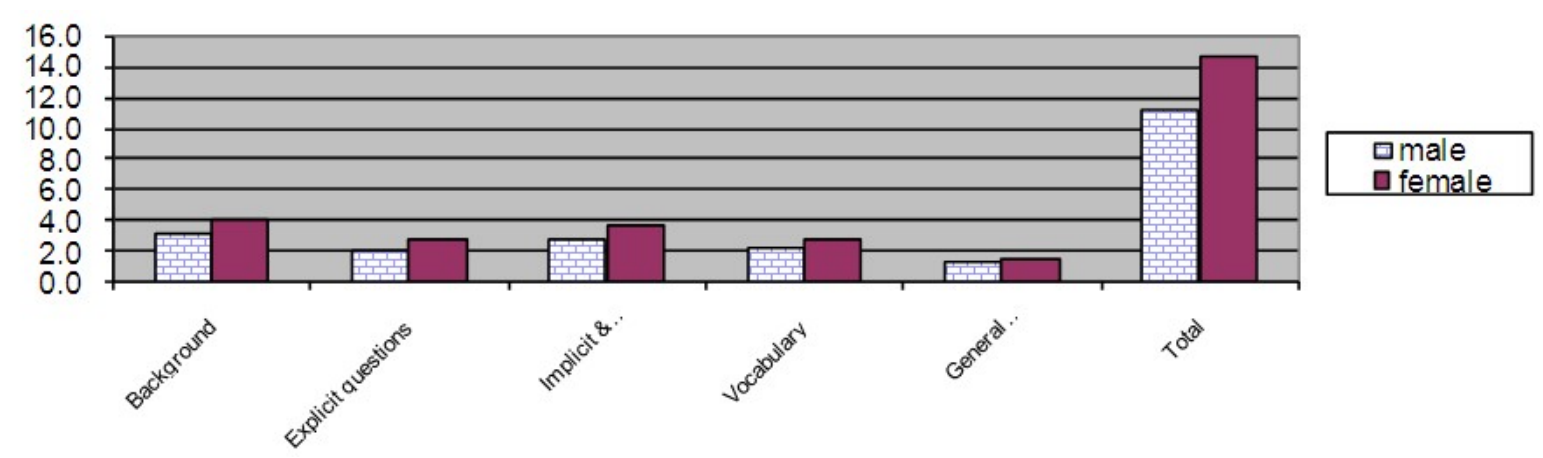

Figure 2. The average performance of the male and female students in EFL reading test

About the questionnaire introduced to the students after doing the test and in order to assess the association between gender and the performance of Sudanese secondary school students in EFL reading, a chi-square test was used to determine whether there were significant differences between the expected frequencies and the observed ones as shown in Tables 7 and 8.

Table 7. The expected and the observed frequencies of the questionnaire (bottom-up statements)

\begin{tabular}{|c|c|c|c|c|c|c|c|c|}
\hline Statement & Sex & Count & $\begin{array}{l}\text { Strongly } \\
\text { Disagree }\end{array}$ & Disagree & Somewhat & Agree & $\begin{array}{l}\text { Strongly } \\
\text { Agree }\end{array}$ & Total \\
\hline \multirow{4}{*}{$\begin{array}{l}\text { Bottom-up } \\
\text { S1 }\end{array}$} & \multirow{2}{*}{ Male } & Observed Count & 12 & 35 & 48 & 42 & 23 & 160 \\
\hline & & Expected Count & 9.1 & 29.9 & 49.6 & 46.4 & 25.1 & 160.0 \\
\hline & \multirow{2}{*}{ Female } & Observed Count & 5 & 21 & 45 & 45 & 24 & 140 \\
\hline & & Expected Count & 7.9 & 26.1 & 43.4 & 40.6 & 21.9 & 140.0 \\
\hline \multirow{4}{*}{$\begin{array}{l}\text { Bottom-up } \\
\text { S2 }\end{array}$} & \multirow{2}{*}{ Male } & Observed Count & 10 & 28 & 51 & 48 & 23 & 160 \\
\hline & & Expected Count & 8.5 & 27.2 & 53.3 & 48.5 & 22.4 & 160.0 \\
\hline & \multirow{2}{*}{ Female } & Observed Count & 6 & 23 & 49 & 43 & 19 & 140 \\
\hline & & Expected Count & 7.5 & 23.8 & 46.7 & 42.5 & 19.6 & 140.0 \\
\hline \multirow{4}{*}{$\begin{array}{l}\text { Bottom-up } \\
\text { S3 }\end{array}$} & \multirow{2}{*}{ Male } & Observed Count & 8 & 23 & 59 & 50 & 20 & 160 \\
\hline & & Expected Count & 7.5 & 22.9 & 58.1 & 50.1 & 20.8 & 160.0 \\
\hline & \multirow{2}{*}{ Female } & Observed Count & 6 & 20 & 50 & 44 & 19 & 140 \\
\hline & & Expected Count & 6.5 & 20.1 & 50.9 & 43.9 & 18.2 & 140.0 \\
\hline \multirow{4}{*}{$\begin{array}{l}\text { Bottom-up } \\
\text { S4 }\end{array}$} & \multirow{2}{*}{ Male } & Observed Count & 22 & 48 & 54 & 26 & 10 & 160 \\
\hline & & Expected Count & 23.5 & 50.7 & 54.9 & 21.9 & 9.1 & 160.0 \\
\hline & \multirow{2}{*}{ Female } & Observed Count & 22 & 47 & 49 & 15 & 7 & 140 \\
\hline & & Expected Count & 20.5 & 44.3 & 48.1 & 19.1 & 7.9 & 140.0 \\
\hline \multirow{4}{*}{$\begin{array}{l}\text { Bottom-up } \\
\text { S5 }\end{array}$} & \multirow{2}{*}{ Male } & Observed Count & 8 & 39 & 70 & 36 & 7 & 160 \\
\hline & & Expected Count & 8.5 & 38.9 & 67.2 & 35.7 & 9.6 & 160.0 \\
\hline & \multirow{2}{*}{ Female } & Observed Count & 8 & 34 & 56 & 31 & 11 & 140 \\
\hline & & Expected Count & 7.5 & 34.1 & 58.8 & 31.3 & 8.4 & 140.0 \\
\hline \multirow{4}{*}{$\begin{array}{l}\text { Bottom-up } \\
\text { S6 }\end{array}$} & \multirow{2}{*}{ Male } & Observed Count & 19 & 56 & 60 & 19 & 6 & 160 \\
\hline & & Expected Count & 25.6 & 58.7 & 50.7 & 19.7 & 5.3 & 160.0 \\
\hline & \multirow{2}{*}{ Female } & Observed Count & 29 & 54 & 35 & 18 & 4 & 140 \\
\hline & & Expected Count & 22.4 & 51.3 & 44.3 & 17.3 & 4.7 & 140.0 \\
\hline \multirow{4}{*}{$\begin{array}{l}\text { Bottom-up } \\
\text { S7 }\end{array}$} & \multirow{2}{*}{ Male } & Observed Count & 6 & 37 & 55 & 45 & 17 & 160 \\
\hline & & Expected Count & 6.4 & 38.9 & 48.0 & 46.9 & 19.7 & 160.0 \\
\hline & \multirow{2}{*}{ Female } & Observed Count & 6 & 36 & 35 & 43 & 20 & 140 \\
\hline & & Expected Count & 5.6 & 34.1 & 42.0 & 41.1 & 17.3 & 140.0 \\
\hline
\end{tabular}


Table 8. The expected and the observed frequencies of the questionnaire (top-down statements)

\begin{tabular}{|c|c|c|c|c|c|c|c|c|}
\hline Statement & Sex & Count & $\begin{array}{l}\text { Strongly } \\
\text { Disagree }\end{array}$ & Disagree & Somewhat & Agree & $\begin{array}{l}\text { Strongly } \\
\text { Agree }\end{array}$ & Total \\
\hline \multirow{4}{*}{$\begin{array}{l}\text { Top-down } \\
\text { S8 }\end{array}$} & \multirow{2}{*}{ Male } & Observed Count & 7 & 32 & 63 & 38 & 20 & 160 \\
\hline & & Expected Count & 6.9 & 28.8 & 61.9 & 40.5 & 21.9 & 160.0 \\
\hline & \multirow{2}{*}{ Female } & Observed Count & 6 & 22 & 53 & 38 & 21 & 140 \\
\hline & & Expected Count & 6.1 & 25.2 & 54.1 & 35.5 & 19.1 & 140.0 \\
\hline \multirow{4}{*}{$\begin{array}{l}\text { Top-down } \\
\text { S9 }\end{array}$} & \multirow{2}{*}{ Male } & Observed Count & 4 & 26 & 61 & 53 & 16 & 160 \\
\hline & & Expected Count & 7.5 & 28.8 & 58.7 & 47.5 & 17.6 & 160.0 \\
\hline & \multirow{2}{*}{ Female } & Observed Count & 10 & 28 & 49 & 36 & 17 & 140 \\
\hline & & Expected Count & 6.5 & 25.2 & 51.3 & 41.5 & 15.4 & 140.0 \\
\hline \multirow{4}{*}{$\begin{array}{l}\text { Top-down } \\
\text { S10 }\end{array}$} & \multirow{2}{*}{ Male } & Observed Count & 14 & 34 & 63 & 33 & 16 & 160 \\
\hline & & Expected Count & 14.4 & 30.9 & 62.9 & 36.8 & 14.9 & 160.0 \\
\hline & \multirow{2}{*}{ Female } & Observed Count & 13 & 24 & 55 & 36 & 12 & 140 \\
\hline & & Expected Count & 12.6 & 27.1 & 55.1 & 32.2 & 13.1 & 140.0 \\
\hline \multirow{4}{*}{$\begin{array}{l}\text { Top-down } \\
\text { S11 }\end{array}$} & \multirow{2}{*}{ Male } & Observed Count & 8 & 35 & 56 & 42 & 19 & 160 \\
\hline & & Expected Count & 10.7 & 36.8 & 54.4 & 40.5 & 17.6 & 160.0 \\
\hline & \multirow{2}{*}{ Female } & Observed Count & 12 & 34 & 46 & 34 & 14 & 140 \\
\hline & & Expected Count & 9.3 & 32.2 & 47.6 & 35.5 & 15.4 & 140.0 \\
\hline \multirow{4}{*}{$\begin{array}{l}\text { Top-down } \\
\text { S12 }\end{array}$} & \multirow{2}{*}{ Male } & Observed Count & 12 & 41 & 58 & 36 & 13 & 160 \\
\hline & & Expected Count & 10.1 & 42.1 & 54.4 & 38.9 & 14.4 & 160.0 \\
\hline & \multirow{2}{*}{ Female } & Observed Count & 7 & 38 & 44 & 37 & 14 & 140 \\
\hline & & Expected Count & 8.9 & 36.9 & 47.6 & 34.1 & 12.6 & 140.0 \\
\hline \multirow{4}{*}{$\begin{array}{l}\text { Top-down } \\
\text { S13 }\end{array}$} & \multirow{2}{*}{ Male } & Observed Count & 5 & 40 & 58 & 32 & 25 & 160 \\
\hline & & Expected Count & 4.8 & 36.8 & 56.5 & 35.2 & 26.7 & 160.0 \\
\hline & \multirow{2}{*}{ Female } & Observed Count & 4 & 29 & 48 & 34 & 25 & 140 \\
\hline & & Expected Count & 4.2 & 32.2 & 49.5 & 30.8 & 23.3 & 140.0 \\
\hline \multirow{4}{*}{$\begin{array}{l}\text { Top-down } \\
\text { S14 }\end{array}$} & \multirow{2}{*}{ Male } & Observed Count & 12 & 35 & 62 & 27 & 24 & 160 \\
\hline & & Expected Count & 10.7 & 34.1 & 62.9 & 29.3 & 22.9 & 160.0 \\
\hline & \multirow{2}{*}{ Female } & Observed Count & 8 & 29 & 56 & 28 & 19 & 140 \\
\hline & & Expected Count & 9.3 & 29.9 & 55.1 & 25.7 & 20.1 & 140.0 \\
\hline \multirow{4}{*}{$\begin{array}{l}\text { Top-down } \\
\text { S15 }\end{array}$} & Mole & Observed Count & 13 & 33 & 67 & 25 & 22 & 160 \\
\hline & Vidale & Expected Count & 14.4 & 34.7 & 65.6 & 26.1 & 19.2 & 160.0 \\
\hline & Female & Observed Count & 14 & 32 & 56 & 24 & 14 & 140 \\
\hline & remale & Expected Count & 12.6 & 30.3 & 57.4 & 22.9 & 16.8 & 140.0 \\
\hline & Male & Observed Count & 12 & 32 & 52 & 46 & 18 & 160 \\
\hline Top-down & Virale & Expected Count & 10.7 & 36.8 & 53.3 & 43.2 & 16.0 & 160.0 \\
\hline S16 & & Observed Count & 8 & 37 & 48 & 35 & 12 & 140 \\
\hline & Female & Expected Count & 9.3 & 32.2 & 46.7 & 37.8 & 14.0 & 140.0 \\
\hline & Male & Observed Count & 18 & 36 & 53 & 39 & 14 & 160 \\
\hline Top-down & Mrale & Expected Count & 18.7 & 25.6 & 56.0 & 37.3 & 22.4 & 160.0 \\
\hline S17 & & Observed Count & 17 & 12 & 52 & 31 & 28 & 140 \\
\hline & Female & Expected Count & 16.3 & 22.4 & 49.0 & 32.7 & 19.6 & 140.0 \\
\hline & & Observed Count & 37 & 22 & 56 & 31 & 14 & 160 \\
\hline Top-down & Male & Expected Count & 34.1 & 18.7 & 59.2 & 30.4 & 17.6 & 160.0 \\
\hline & $\mathrm{Fe}$ & Observed Count & 27 & 13 & 55 & 26 & 19 & 140 \\
\hline & remide & Expected Count & 29.9 & 16.3 & 51.8 & 26.6 & 15.4 & 140.0 \\
\hline Top-down & Male & Observed Count & 20 & 37 & 54 & 34 & 15 & 160 \\
\hline
\end{tabular}




\begin{tabular}{|c|c|c|c|c|c|c|c|c|}
\hline \multirow[t]{3}{*}{ S19 } & & Expected Count & 23.5 & 35.7 & 54.9 & 30.4 & 15.5 & 160.0 \\
\hline & \multirow{2}{*}{ Female } & Observed Count & 24 & 30 & 49 & 23 & 14 & 140 \\
\hline & & Expected Count & 20.5 & 31.3 & 48.1 & 26.6 & 13.5 & 140.0 \\
\hline \multirow{4}{*}{$\begin{array}{l}\text { Top-down } \\
\text { S20 }\end{array}$} & \multirow{2}{*}{ Male } & Observed Count & 39 & 28 & 56 & 21 & 16 & 160 \\
\hline & & Expected Count & 36.3 & 33.6 & 54.4 & 22.4 & 13.3 & 160.0 \\
\hline & \multirow{2}{*}{ Female } & Observed Count & 29 & 35 & 46 & 21 & 9 & 140 \\
\hline & & Expected Count & 31.7 & 29.4 & 47.6 & 19.6 & 11.7 & 140.0 \\
\hline \multirow{4}{*}{$\begin{array}{l}\text { Top-down } \\
\text { S21 }\end{array}$} & \multirow{2}{*}{ Male } & Observed Count & 38 & 34 & 49 & 21 & 18 & 160 \\
\hline & & Expected Count & 36.8 & 34.1 & 50.1 & 20.8 & 18.1 & 160.0 \\
\hline & \multirow{2}{*}{ Female } & Observed Count & 31 & 30 & 45 & 18 & 16 & 140 \\
\hline & & Expected Count & 32.2 & 29.9 & 43.9 & 18.2 & 15.9 & 140.0 \\
\hline \multirow{4}{*}{$\begin{array}{l}\text { Top-down } \\
\text { S22 }\end{array}$} & \multirow{2}{*}{ Male } & Observed Count & 31 & 34 & 52 & 23 & 20 & 160 \\
\hline & & Expected Count & 31.5 & 34.1 & 53.3 & 23.5 & 17.6 & 160.0 \\
\hline & \multirow{2}{*}{ Female } & Observed Count & 28 & 30 & 48 & 21 & 13 & 140 \\
\hline & & Expected Count & 27.5 & 29.9 & 46.7 & 20.5 & 15.4 & 140.0 \\
\hline
\end{tabular}

When looking at Tables 7 and 8 to the observed account and the expected count in order to see the association of gender with other variables, it is obvious that the observed differences between them were not enough to be significant. When going down to the Chi-square test Tables 9 and 10, the percentages in all bottom-up and top-down items were less than $20 \%$. Since these percentages were less, then this assumption was not violated. In all the items except item no. 17, Pearson Chi-square values were not P-values. They were all greater than the alpha value 0.05 , so this revealed that their results were not statistically significant and hence the ultimate hypothesis, which said there was no significant association between gender and the performances of Sudanese secondary school students in EFL reading, was accepted. In other words, students' performances were not dependent from gender. They were independent.

Table 9. Chi-square test (bottom-up statements)

\begin{tabular}{|c|c|c|c|c|}
\hline Statement & Test & Value & $\mathrm{df}$ & Asymp. Sig (2-sided) \\
\hline \multirow[t]{4}{*}{ Bottom-up S1 } & Pearson Chi-square & $5.294 \mathrm{~A}$ & 4 & 0.258 \\
\hline & Likelihood Ratio & 5.395 & 4 & 0.249 \\
\hline & Linear-by-Linear Association & 4.114 & 1 & 0.043 \\
\hline & \multicolumn{4}{|c|}{ a. 0 cells $(.0 \%)$ have expected count less than 5 . The minimum expected count is 7.93 . } \\
\hline \multirow[t]{4}{*}{ Bottom-up S2 } & Pearson Chi-square & $0.856 \mathrm{~A}$ & 4 & 0.931 \\
\hline & Likelihood Ratio & 0.864 & 4 & 0.930 \\
\hline & Linear-by-Linear Association & 0.109 & 1 & 0.741 \\
\hline & \multicolumn{4}{|c|}{ a. 0 cells $(.0 \%)$ have expected count less than 5 . The minimum expected count is 7.47. } \\
\hline \multirow[t]{4}{*}{ Bottom-up S3 } & Pearson Chi-square & $1.319 \mathrm{~A}$ & 5 & 0.933 \\
\hline & Likelihood Ratio & 1.701 & 5 & 0.889 \\
\hline & Linear-by-Linear Association & 0.248 & 1 & 0.618 \\
\hline & \multicolumn{4}{|c|}{ a. 2 cells $(16.7 \%)$ have expected count less than 5 . The minimum expected count is 0.47 . } \\
\hline \multirow[t]{4}{*}{ Bottom-up S4 } & Pearson Chi-square & $2.411 \mathrm{~A}$ & 4 & 0.661 \\
\hline & Likelihood Ratio & 2.439 & 4 & 0.656 \\
\hline & Linear-by-Linear Association & 1.580 & 1 & 0.209 \\
\hline & \multicolumn{4}{|c|}{ a. 0 cells $(.0 \%)$ have expected count less than 5 . The minimum expected count is 7.93 . } \\
\hline \multirow[t]{3}{*}{ Bottom-up S5 } & Pearson Chi-square & $1.835 \mathrm{~A}$ & 4 & 0.766 \\
\hline & Likelihood Ratio & 1.837 & 4 & 0.766 \\
\hline & Linear-by-Linear Association & 0.224 & 1 & 0.636 \\
\hline
\end{tabular}




\begin{tabular}{llccc}
\hline \multirow{4}{*}{ Bottom-up S6 } & \multicolumn{4}{l}{ a. 0 cells (.0\%) have expected count less than 5. The minimum expected count is 7.47. } \\
& Pearson Chi-square & $7.827 \mathrm{~A}$ & 4 & 0.098 \\
& Likelihood Ratio & 7.888 & 4 & 0.096 \\
& Linear-by-Linear Association & 3.560 & 1 & 0.059 \\
& a. 0 cells (1\%) have expected count less than & 5. & The minimum expected count is 4.67. \\
Top-down S7 & Pearson Chi-square & $3.429 \mathrm{~A}$ & 4 & 0.489 \\
& Likelihood Ratio & 3.450 & 4 & 0.486 \\
& Linear-by-Linear Association & 0.254 & 1 & 0.614 \\
& a. 0 cells (.0\%) have expected count less than 5. The minimum expected count is 5.60. \\
\hline
\end{tabular}

Table 10. Chi-square test (top-down statements)

\begin{tabular}{|c|c|c|c|c|}
\hline Statement & Test & Value & df & Asymp. Sig (2-sided) \\
\hline \multirow[t]{4}{*}{ Top-down S8 } & Pearson Chi-square & $1.489 \mathrm{~A}$ & 4 & 0.829 \\
\hline & Likelihood Ratio & 1.493 & 4 & 0.828 \\
\hline & Linear-by-Linear Association & 1.133 & 1 & 0.287 \\
\hline & \multicolumn{4}{|c|}{ a. 0 cells $(.0 \%)$ have expected count less than 5 . The minimum expected count is 6.07 . } \\
\hline \multirow[t]{4}{*}{ Top-down S9 } & Pearson Chi-square & $5.925 \mathrm{~A}$ & 4 & 0.205 \\
\hline & Likelihood Ratio & 6.006 & 4 & 0.199 \\
\hline & Linear-by-Linear Association & 1.861 & 1 & 0.172 \\
\hline & \multicolumn{4}{|c|}{ a. 0 cells $(.0 \%)$ have expected count less than 5 . The minimum expected count is 6.53 . } \\
\hline \multirow[t]{4}{*}{ Top-down S10 } & Pearson Chi-square & $1.680 \mathrm{~A}$ & 4 & 0.794 \\
\hline & Likelihood Ratio & 1.682 & 4 & 0.794 \\
\hline & Linear-by-Linear Association & 0.179 & 1 & 0.672 \\
\hline & \multicolumn{4}{|c|}{ a. 0 cells $(.0 \%)$ have expected count less than 5 . The minimum expected count is 12.60 . } \\
\hline \multirow[t]{4}{*}{ Top-down S11 } & Pearson Chi-square & $2.070 \mathrm{~A}$ & 4 & 0.723 \\
\hline & Likelihood Ratio & 2.072 & 4 & 0.723 \\
\hline & Linear-by-Linear Association & 1.473 & 1 & 0.225 \\
\hline & \multicolumn{4}{|c|}{ a. 0 cells $(.0 \%)$ have expected count less than 5 . The minimum expected count is 9.33 . } \\
\hline \multirow[t]{4}{*}{ Top-down S12 } & Pearson Chi-square & $2.078 \mathrm{~A}$ & 4 & 0.721 \\
\hline & Likelihood Ratio & 2.089 & 4 & 0.719 \\
\hline & Linear-by-Linear Association & 0.828 & 1 & 0.363 \\
\hline & \multicolumn{4}{|c|}{ a. 0 cells $(.0 \%)$ have expected count less than 5 . The minimum expected count is 8.87 . } \\
\hline \multirow[t]{4}{*}{ Top-down S13 } & Pearson Chi-square & $1.542 \mathrm{~A}$ & 4 & 0.819 \\
\hline & Likelihood Ratio & 1.544 & 4 & 0.819 \\
\hline & Linear-by-Linear Association & 1.174 & 1 & 0.279 \\
\hline & \multicolumn{4}{|c|}{ a. 2 cells $(20 \%)$ have expected count less than 5 . The minimum expected count is 4.20 . } \\
\hline \multirow[t]{4}{*}{ Top-down S14 } & Pearson Chi-square & $1.508 \mathrm{~A}$ & 4 & 0.825 \\
\hline & Likelihood Ratio & 1.516 & 4 & 0.824 \\
\hline & Linear-by-Linear Association & 0.873 & 1 & 0.350 \\
\hline & \multicolumn{4}{|c|}{ a. 0 cells $(.0 \%)$ have expected count less than 5 . The minimum expected count is 12.60 . } \\
\hline \multirow[t]{4}{*}{ Top-down S15 } & Pearson Chi-square & $5.294 \mathrm{~A}$ & 4 & 0.258 \\
\hline & Likelihood Ratio & 5.395 & 4 & 0.249 \\
\hline & Linear-by-Linear Association & 4.114 & 1 & 0.043 \\
\hline & \multicolumn{4}{|c|}{ a. 0 cells $(.0 \%)$ have expected count less than 5 . The minimum expected count is 7.93 . } \\
\hline Top-down S16 & Pearson Chi-square & $2.695 \mathrm{~A}$ & 4 & 0.610 \\
\hline
\end{tabular}




\begin{tabular}{|c|c|c|c|c|}
\hline & Likelihood Ratio & 2.700 & 4 & 0.609 \\
\hline & Linear-by-Linear Association & 0.922 & 1 & 0.337 \\
\hline & \multicolumn{4}{|c|}{ a. 0 cells $(.0 \%)$ have expected count less than 5 . The minimum expected count is 9.33 . } \\
\hline \multirow[t]{4}{*}{ Top-down S17 } & Pearson Chi-square & $16.358 \mathrm{~A}$ & 4 & $0.003 *$ \\
\hline & Likelihood Ratio & 16.935 & 4 & 0.002 \\
\hline & Linear-by-Linear Association & 5.526 & 1 & 0.018 \\
\hline & a. 0 cells $(.0 \%)$ have expected & nt less tha & he 1 & Ium expected count is 16.33 . \\
\hline \multirow[t]{4}{*}{ Top-down S18 } & Pearson Chi-square & $3.765 \mathrm{~A}$ & 4 & 0.439 \\
\hline & Likelihood Ratio & 3.784 & 4 & 0.436 \\
\hline & Linear-by-Linear Association & 2.071 & 1 & 0.150 \\
\hline & a. 0 cells $(.0 \%)$ have expected & nt less tha & he 1 & Ium expected count is 15.40 . \\
\hline \multirow[t]{4}{*}{ Top-down S19 } & Pearson Chi-square & $2.171 \mathrm{~A}$ & 4 & 0.704 \\
\hline & Likelihood Ratio & 2.176 & 4 & 0.703 \\
\hline & Linear-by-Linear Association & 0.677 & 1 & 0.411 \\
\hline & a. 0 cells $(.0 \%)$ have expected & nt less tha & he 1 & um expected count is 13.53 . \\
\hline \multirow[t]{4}{*}{ Top-down S20 } & Pearson Chi-square & $3.873 \mathrm{~A}$ & 4 & 0.424 \\
\hline & Likelihood Ratio & 3.889 & 4 & 0.421 \\
\hline & Linear-by-Linear Association & 0.151 & 1 & 0.698 \\
\hline & a. 0 cells $(.0 \%)$ have expected & nt less tha & he 1 & um expected count is 11.67 . \\
\hline \multirow[t]{4}{*}{ Top-down S21 } & Pearson Chi-square & $0.146 \mathrm{~A}$ & 4 & 0.997 \\
\hline & Likelihood Ratio & 0.146 & 4 & 0.997 \\
\hline & Linear-by-Linear Association & 0.045 & 1 & 0.832 \\
\hline & a. 0 cells $(.0 \%)$ have expected & nt less tha & he 1 & um expected count is 15.87 . \\
\hline \multirow[t]{4}{*}{ Top-down S22 } & Pearson Chi-square & $0.809 \mathrm{~A}$ & 4 & 0.937 \\
\hline & Likelihood Ratio & 0.816 & 4 & 0.936 \\
\hline & Linear-by-Linear Association & 0.255 & 1 & 0.614 \\
\hline & \multicolumn{4}{|c|}{ a. 0 cells $(.0 \%)$ have expected count less than 5 . The minimum expected count is 15.40 . } \\
\hline
\end{tabular}

\section{Conclusion}

Depending on the results of the study and data analysis, it seems reasonable to conclude that in teaching Sudanese EFL secondary schools, it is necessary to adopt the interactive method of reading which integrates elements of both levels of processing skills: bottom-up and top-down. It is important to provide students explicit instruction of some lower level processing skills (bottom-up) such as teaching students some strategies in phonemic awareness, word recognition, and syntactic analysis, and some higher-level of processing skills (top-down) such as teaching students some strategies in guessing, inferences, and predicting. Good applications of techniques and procedures of teaching EFL reading may prove to be a viable intervention for improving students' performance in EFL reading.

\section{Recommendations}

Bearing in mind the conclusions derived from the study, these points are recommended to any teacher of EFL reading:

1) The objectives of teaching English language in secondary schools should make an obvious plan to promote teaching EFL reading. Ambiguity leads to a weak output. In preparing and developing a reading syllabus, it is important to consider the balance between top-down and bottom-up levels of processing.

2) When teaching EFL reading, combining approaches to reading is recommended in order to train students to become efficient, effective and independent readers. 
3) Teachers have to provide learners with appropriate skills and strategies needed to become strategic readers in particular and strategic learners in general. They have to arrange different activities help students to acquire and develop competences and be involved in the teaching-learning process.

4) Developing fluency in EFL readers requires training in both levels of processes top-down and bottom-up.

5) Encouraging extensive reading and reading for pleasure.

\section{References}

Aebersold, J. A., \& Field, M. L. (1997). From reader to reading teacher. Cambridge: Cambridge University Press.

Anderson. R. C., \& Pearson, P. D. (1984). A schema-theoretic view of basic processes in reading comprehension. In P. D. Pearson (Ed.), Handbook of Reading Research (pp. 255-292). New York: Longman.

Bamford, J., \& Day, R. R. (1998). Extensive Reading in the Second Language Classroom. Cambridge, UK: Cambridge University Press.

Barnett, M. A. (1988). Reading through context: How real and perceived strategy affects L2 comprehension. The Modern Language Journal, 72(2), 150-162. http://dx.doi.org/10.1111/j.1540-4781.1988.tb04177.x

Carrel, P. L. (1988). Interactive text processing: implications for ESL/second language reading classrooms. In P. Carrel, J. Devine, \& D. Eskey (Eds.), Interactive approaches to second language reading (pp. 239-259). http://dx.doi.org/10.1017/CBO9781139524513.023

Carrell, P. L. (1989). Metacognitive awareness and second language reading. TESOL Quarterly, 73, 121-134. http://dx.doi.org/10.2307/326568

Crystal, D. (1987). The Cambridge Encyclopedia of Language. Cambridge: Cambridge university press.

Eskey, D. E. (1988). Holding in the bottom: An interactive approach to the language problems of second language. In P. Carrell, J. Devine, \& D. Eskey (Eds.), Interactive approaches to second language reading (pp. 93-100). New York: Cambridge.

Fromkin, V., \& Rodman, R. (1983). An introduction to language. New York, NY: Holt Saunders.

Gagne, E. D., Yokovich, C. W., \& Yokovich, F. R. (1993). The cognitive psychology of school learning (2nd ed.). New York: Harper Collins.

Goodman, K. S. (1967). "Reading: A Psycholinguistic Guessing Game”. Journal of the Reading Specialist (May 1967), 126-135. http://dx.doi.org/10.1080/19388076709556976

Mikulecky, B. S. (1990). A Short Course in Teaching Reading Skills. Reading, MA: Addison-Wesley Publishing Co.

Nation, I. S. P. (2009). Teaching ESL/EFL Reading and Writing. New York: Rout ledge.

Nuttal, C. (1983). Teaching Reading Skills in a Foreign Language. Oxford, UK: Heinemann Publishers Ltd.

Rumelhart, D. E. (1977). Toward an interactive model for reading. In W. Otto (Ed.), Reading problems. Boston, MA: Addison-Wesley.

Sun-Young, Oh. (2001). Two types of Input Modification and EFL Reading Comprehension: Simplification versus Elaboration. Teachers of Speakers of Other Languages, Inc. TESOL Quarterly, 35(1), 69-96. http://dx.doi.org/10.2307/3587860

\section{Note}

Note 1. SPINE: Sudan Practical Integrated National English Series. 


\section{Appendix A}

\section{The Comprehension Test}

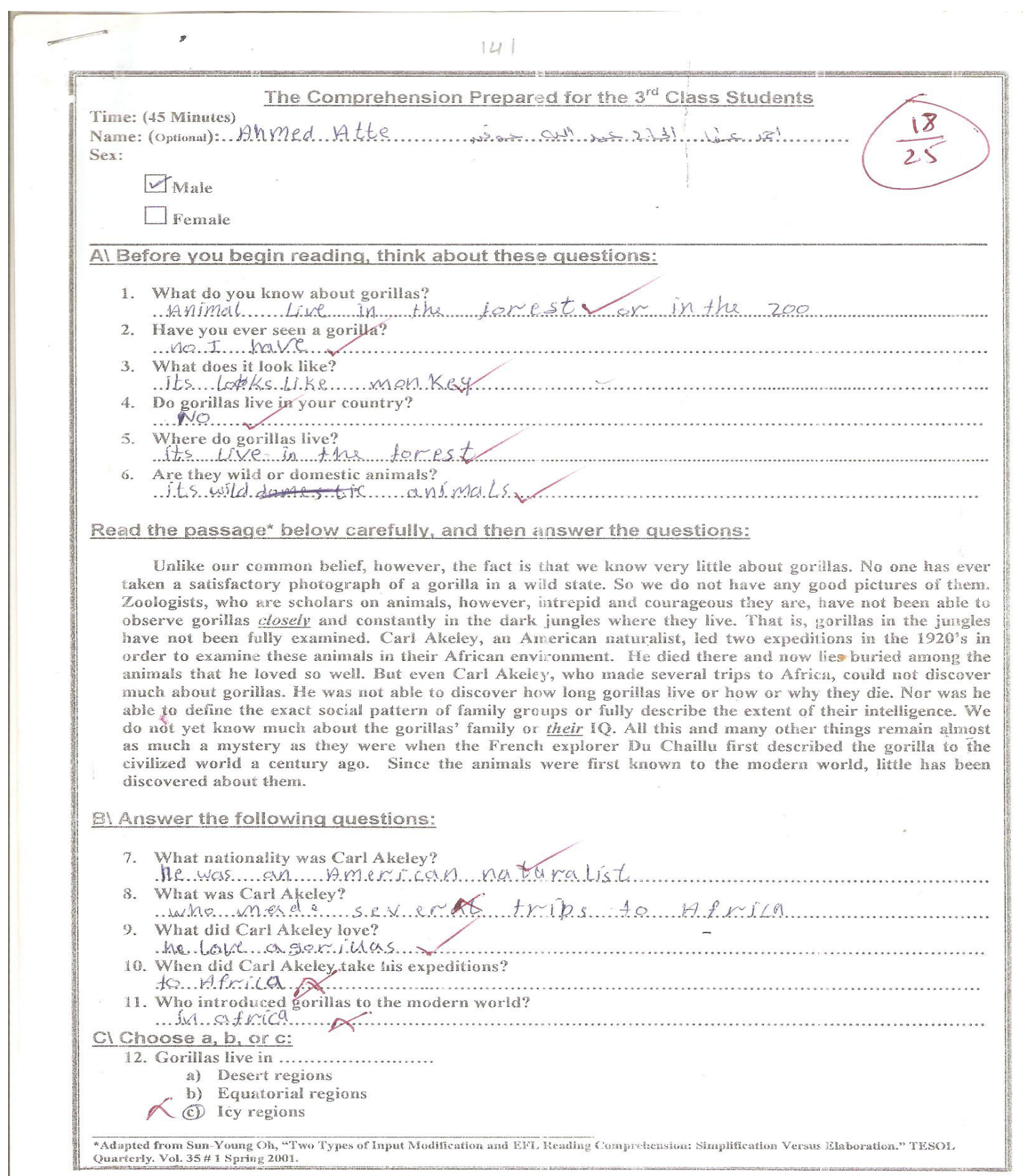


13. Gorillas feed on
(a) fruits
b) meet
c) vegetables

14. To study gorillas, Carl Akeley made two expeditions to

a) Asia

(b) Africa

c) America

15. Carl Akeley died in
a) America
b) Australia
(c) Africa

16. Du Chaillu described gorillas in the

a) Seventeen hundreds

b) Eighteen hundreds

(c) Nineteen hundreds

17. The best title for this passage is
a) Carl Akeley
b) Du Chaillu
(c) Gorillas

Dl Choose $a, b$, or $c$ :

18. Another word for "jungles' is

a) caves

b) gardens

(c) forests

19. The opposite of the underlined word 'closely' in line 3 is

$$
\begin{aligned}
& \text { (ai) nearly } \\
& \text { b) distant } \\
& \text { c) directly }
\end{aligned}
$$

20. A 'naturalist' is

a) a pharmacist

(b) a zoologist

c) a chemist

21. An 'expedition' is

a) a disease

b) a kind of animals

(c) a scientific journey

22. The pronoun 'their' in line 9 refers to
(di) gorillas
b) trips
c) scholars

\section{EIPut [I] for true or [F] for false:}

23. Now gorillas are well -known all over the world. $/ x$

24. Carl Akeley and Du Chaillu did research about dark jungles in Africa.

25. The main aim of the two scientists was to expand the world's knowledge about gorillas.

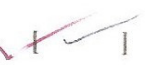




\section{Appendix B}

The Questionnaire

Dear student,

You, with genuine answers, will contribute much in promoting teaching EFL in the Sudan, through answering this questionnaire. The answers will be analyzed confidentially. I would like to reassure you that they would be used for scientific purposes only.

Thanks,

Part 1. Personal Identification

First, please provide some information about yourself. Please tick $(\checkmark)$ the appropriate space(s)

1) Name (Optional):

2) Sex:

Male

Female

3) School:

Part 2. Please tick $(\checkmark)$ the appropriate space:

The following statements are about strategies used by EFL students in determining unfamiliar words. Please indicate your level of agreement or disagreement with each statement by ticking the appropriate space: (1) indicates the strongest agreement, (5) indicates strong disagreement.

Table B1.The questionnaire (bottom-up statements)

\begin{tabular}{llllll}
\hline \multirow{2}{*}{ Statement } & Strongly Agree & Agree & Somewhat & Disagree & Strongly Disagree \\
\cline { 2 - 6 } & 4 & 3 & 2 & 1 & 0 \\
\hline
\end{tabular}

Part A: Bottom-up Strategies

1. I have phonemic awareness of words and phrases in the text.

2. I attempt to understand the meanings of individual words.

3. I try to understand the meaning or structure of a clause or a sentence.

4. I restate the content by paraphrasing or rereading.

5. I am able to identify the grammatical categories of words.

6. I am able to identify the meaning of words and phrases.

7. I infer the meaning of an unknown word through retention. 
Table B2. The questionnaire (top-down statements)

\begin{tabular}{llllll}
\hline \multirow{2}{*}{ Statement } & Strongly Agree & Agree & Somewhat & Disagree & Strongly Disagree \\
\cline { 2 - 6 } & 4 & 3 & 2 & 1 & 0 \\
\hline
\end{tabular}

Part B: Top-down Strategies

8. I use my prior knowledge and experience about the content of the text.

9. I form an image about whole or some portions of the text.

10. I use imagination and association as techniques to link the meanings together.

11. I scan and skim the passage for a general understanding.

12. I predict the likely content of the succeeding portions of the text.

13. I confirm, modify or reject the prediction I have made about the succeeding portions of the text.

14. I connect the new information with the previously stated content.

15. I benefit from the textual clues in the text to anticipate information.

16. I make sense of what I read.

17. I distinguish between important information and details.

18. I am able to notice inconsistences in the text.

19. I employ strategies to make inconsistences understandable.

20. I comment on the significance of the content.

21. I summarize the whole or some portion(s) of the text.

22. I make inferences or draw conclusions about the content of the text.

\section{Copyrights}

Copyright for this article is retained by the author(s), with first publication rights granted to the journal.

This is an open-access article distributed under the terms and conditions of the Creative Commons Attribution license (http://creativecommons.org/licenses/by/3.0/). 